Neue Erstattungsbeträge

\title{
Gliptine bleiben weiter im Angebot
}

\begin{abstract}
Nach der zweiten Nutzenbewertung erfolgte die Einigung auf neue Erstattungsbeträge. Die etwa 1,5 Millionen Diabetiker, die mit Sitapgliptin und Saxagliptin sowie deren Kombinationen mit Metformin behandelt werden, können diese Arzneien auch zukünftig erhalten. Denn vor Kurzem haben sich die Hersteller mit dem GKV-Spitzenverband auf neue, niedrigere Erstattungsbeträge geeinigt.
\end{abstract}

Hintergrund ist eine zweite Nutzenbewertung für beide Wirkstoffe, die der Bundesaussschuss 2013 mit einer Befristung seiner damaligen Nutzenbewertungsbeschlüsse angeordnet hatte. Dazu hatten die Hersteller die Ergebnisse umfangreicher Studien mit einer bis zu fünfstelligen Zahl von Studienteilnehmern vorgelegt. Das Ergebnis der Auswertung war allerdings ernüchternd.

Für Sitagliptin anerkannte der GBA aufgrund der neuen Studienlage in Kombination mit Metformin einen Anhaltspunkt für geringen Zusatznutzen, und zwar wegen des Vorteils eines geringeren Schadens versus der zweckmäßigen Vergleichstherapie (Gli- benclamid o. Glimepirid plus Metformin). Aufgrund der neuen Studienlage hat der GBA seine Bewertung für die Sitagliptin-Monotherapie korrigiert; er sieht nun keinen Beleg mehr für einen damals noch anerkannten geringen Zusatznutzen. Sitagliptin wird von MSD als Januvia ${ }^{\oplus}$ und Janumet ${ }^{\oplus}$ (Kombi), im Co-Marketing mit Berlin-Chemie als Xelevia ${ }^{\oplus}$ und Velmetia angeboten. Für Saxagliptin plus Metformin wurde der 2013 anerkannte Anhalt für geringen Zusatznutzen durch die neue Studienlage nicht bestätigt. Die Studie SAVOR-TIMI 53 sei durch ihre Größe, Dauer und die Erhebung von patientenrelevanten kardiovaskulären Endpunkten für die Zusatznutzen-Bewertung relevant. Dabei habe sich gezeigt, dass es statistisch signifikante Differenzen zu ungunsten von Saxagliptin/Metformin für den Endpunkt „Hospitalisierung aufgrund von Herzinsuffizienz“ gibt. Saxagliptin wird von AstraZeneca als Onglyza ${ }^{\oplus}$ u. Komboglyze ${ }^{\varpi}$ angeboten.

Die Deutsche Diabetes-Gesellschaft (DDG) hatte diese Bewertungen kritisiert. Die betreffenden Medikamente hätten sich seit 10 Jahren in der Praxis bewährt. Im Vergleich zu Sulfonylharnstoffen hätten sie z.B. den Vorteil, keine Hypoglykämien zu verursachen.

Helmut Laschet

\section{Hier steht eine Anzeige.}

\section{Springer}

